\title{
Effect of Bilateral Hippocampal Destruction on the Acquisition and Extinction of an Operant Response'
}

\author{
LEONARD W. SCHMALTZ AND ROBERT L. ISAACSON \\ Department of Psichology, University of Michigan, Ann Arbor, Michigan 48104
}

(Reccived 6 February 1967)

\begin{abstract}
SCHAALTZ, L. IV. INI) R. L. ISAACSON. Effect of bilateral hippocampal destruction on the acquisition and extinction of an operant response'. Pirssiol. BeHAv. 2 (3) 291-298, 1967. - Rats suffering radical bilateral hippocampal destruction, partial. destruction of the puisteroliteral neocortex (control animals), and unoperated rats were given long experience with CRF (cintinuous reinforcenent) and then shifted to a simple extinction schedule. Twice during the extinction phase of the experiment, the animals were given 30 response-contingent reinforcements. The hippocampectomized subjects were not found to respond more than the other animals on the CRF or extinction schedules; but did, however, show greater increases in their batr press rates after experiencing the 30 reinforced responses. An activity measure was taken of all animals belore and after certain training sessions. Hours of food deprivation were found to differentially affect the ativity kevels of the lesioned and non-lesioned subjects.
\end{abstract}

Hippo:anmus Lxtinction Operant conditioning

NhHol chl beir lates of responsc on continuous reinforcement schedules are not geater than those of control animals, hippocampectomired rats have been found to bar press more than control or unoperated rats on a DRL 20 (differential reinforcement of low rattes of responding) schedule of reinforcement $[1,10,11]$ and on a VI (variable interval) schedule of reinforcement [6].

It was hypothesized by Sichmaltz and Isaacson that hippocampectomized rats performed poorly because they were impaired in their ability to inhibit the bar press response after it had been firmly established. The present study further investigates the ability of hippocampectomized rats to withhold responses. We undertook to determine whether the increased responding found under intermittant schedules, noted above, would be displayed under a sequence of alternated continuous reinforcement and non-reinforcement schedules. Accordingly, animals with posterolateral neocortical destruction, hippocampal damage, and unoperated rats were given considerable training under continuous reinforcement conditions and shifted to non-reinforcement. Interposed in this non-reinforcement period were two periods in which 30 response-contingent reinforcements were provided to determine whether these reinforced responses would have differential effects on subsequent extinclion sessions among the three groups of animals.

An activity measure was taken of all animals before and after certain trauning sessions. Cumulative records were made of their bar presses on CRF and extinction.
METHOD

Subjects were 22 naive male hooded rats approximately 110-days-old at the start of the experiment.

An operant chamber (Grason-Stadler Model E3125A-300) was used which discharged a $45 \mathrm{mg}$ food pellet as a reinforcement.

An ultrasonic device was used for recording activity levels. The apparatus has been described in detail previously [9]. The pulses from this device were used to fire a tube-controlled electromagnetic counter and a cumulative recorder. The chamber used to measure activity was constructed of wood and was 11 in. $\times 23$ in. and 20 in. high. It was placed in a quiet room and was covered with sound shielding material. The transducers were embedded in a side wall 4 in. apart, $9 \mathrm{in}$. from the end of the chamber, and $2.5 \mathrm{in.}$ from the top. The floor and roof of the chamber were also made of wood. One $60 \mathrm{~W}$ bulb positioned in a top corner lighted the chamber.

Previous to surgery, all subjects received 14 days of handling and gentling for $30 \mathrm{~min}$ each day. During this time, they were given ad libitum food and weighed daily.

Subjects were anesthetized with sodium pentobarbital; they were not held in a stereotaxic instrument during the operation. The general operative procedure has been described before [4]. In the experimental group ( 7 animals), the hippocampus was first exposed and removed by aspiration. In making the neocortical lesions (8 animals), the hippocampus was similarly exposed but left intact.

${ }^{1}$ This study was supported in part by a grant from the National Institute of Health to Robert L. Isaacson (NIH MH-11285-01). 
The operated rats were given a 1 month recovery period. During the first 2 weeks after surgery, they were given ad libitum food. The unoperated animals also received ad libitum food during this time.

A 23-hr deprivation schedule was initiated for all animals two weeks before shaping the behavior of the animals to make the bar press response. The rats were allowed to eat mash ad libitum for $1 \mathrm{hr}$ each day. Animals were weighed daily just before feeding. At the end of the 2-week period all animal's weights had stabilized at approximately 90 per cent of their ad libitum weights.

After the shaping had been accomplished in all subjects, they were given 30 consecutive 30 -min sessions under continuous reinforcement (CRF). The number of bar presses per session was recorded for each animal. One hr after each CRF session, the animals were allowed to eat mash ad libitum for $1 \mathrm{hr}$. They were then deprived $21.5 \mathrm{hr}$ until the next CRF session. This $21.5 \mathrm{hr}$ deprivation schedule was maintained throughout the experiment. Subjects were weighed each day before being placed in the operant chamber.

On day 31 , the animals were switched to simple extinction. A series of 15 consecutive daily 30 -min extinction sessions followed the original session. The number of bar presses for each subject was recorded for each session.

One pellet was discharged into the food cup on day 47 before the animal was placed in the operant chamber. The animals were then allowed $\mathbf{3 0}$ response-contingent reinforce-
IIISTOLOGICAL EVALUATION

Following the experiment, all lesioned animals were sacrificed by an overdose of sodium pentobarbital, intracardially perfused with 10 per cent formalin, and the brains removed. Following infiltration and embedding with paraffin, serial sections between 15-20 $\mu$ thickness were made throughout the lesioned areas. As a general procedure, every teith section was retained, stained with thionin, and mounted.

The lesion produced in each brain was reconstructed on representative diagrams of the rat brain adapted from tho atlas by DeGroot [2] at regularly spaced levels throughout the lesioned area. Superimposed on these representative diagrams were $0.5 \mathrm{~cm}$ squares. By counting the number of squares covered by each lesion, it was possible to compute a numerical index of the amount of total brain damage sustained by the animal and the extent of damage to specific structures within the brain.

In addition, drawings of the maximal and minimal extent of the lesions were prepared for the hippocampal and neodecorticate animals from the individual reconstructions. In so doing the largest lesion on each side of the brain at each of the seven representative sections and the smallest lesion on each side of the brain at the same levels were combined onto the same drawings. These are presented in Figs. 1 and 2.

In general, brain damage in the lesioned animals was limited to five structures-the posterior neocortex, the corpus callosum, the fimbria, the hippocampus, and the most

TABLE 1

I) Mmal Sustalned by the Lesioned Animals

\begin{tabular}{|c|c|c|c|c|c|c|}
\hline \multirow[b]{2}{*}{ Group } & \multicolumn{6}{|c|}{ Mean units $(0.5 \mathrm{~cm}$ squares $)$ of damage* } \\
\hline & Neocortex & $\begin{array}{l}\text { Corpus } \\
\text { callosum }\end{array}$ & Fimbria & Hippocampus & $\begin{array}{l}\text { Caudate } \\
\text { nucleus }\end{array}$ & Total \\
\hline $\begin{array}{l}\text { Hippocampal } \\
\text { destruction }\end{array}$ & 1280.1 & 361.4 & 66.4 & 665.3 & 8.7 & 2382.0 \\
\hline $\begin{array}{l}\text { Cirtical } \\
\text { destruction }\end{array}$ & 1140.9 & 250.5 & - & - & 5.2 & 1398.8 \\
\hline $\begin{array}{l}\text { Maximum } \\
\text { possible } \\
\text { damage }\end{array}$ & 1633.0 & 385.0 & 71.0 & 751.0 & 145.0 & 2985.0 \\
\hline
\end{tabular}

ments before the 30-min extinction session. The latency to first bar press and the time required to obtain the 30 reinforcements were recorded for each animal. The subjects were given nine additional extinction sessions.

On day 57, the same procedure was followed as on day 47 . Following this, the rats were given a final nine extinction sessions.

Starting on day 28 and until the completion of the study, cumulative recordings were made of all animals' bar presses for each session.

On days 28-33, each animal was placed in the activity chamber for $30 \mathrm{~min}, 1 \mathrm{hr}$ before being placed in the operant chamber. The animals were given an additional $30 \mathrm{~min}$ in the activity chamber $3 \mathrm{hr}$ after they had eaten on these same days. posterior aspects of the caudate nucleus. Table 1 summarizes the mean units $(0.5 \mathrm{~cm}$ squares) of brain damage sustained by the two groups of animals.

Four of the eight cortical control animals and three of the hippocampectomized rats sustained caudate nucleus damage. In no case was it extensive and a comparison of the behavior of the animals with and without caudate damage did not suggest any effect of the damage within either group.

Regions of gliosis were observed in the thalamus in a number of animals in both the hippocampally ablated and the neodecorticate groups. This involved the lateral nucleus primarily. The gliosis was small in all cases and was not, as fat as could be determined, caused by direct damage to the thalamus. Most likely it resulted from retrograle degeneration or from surgically induced infarction of blood vessels in 

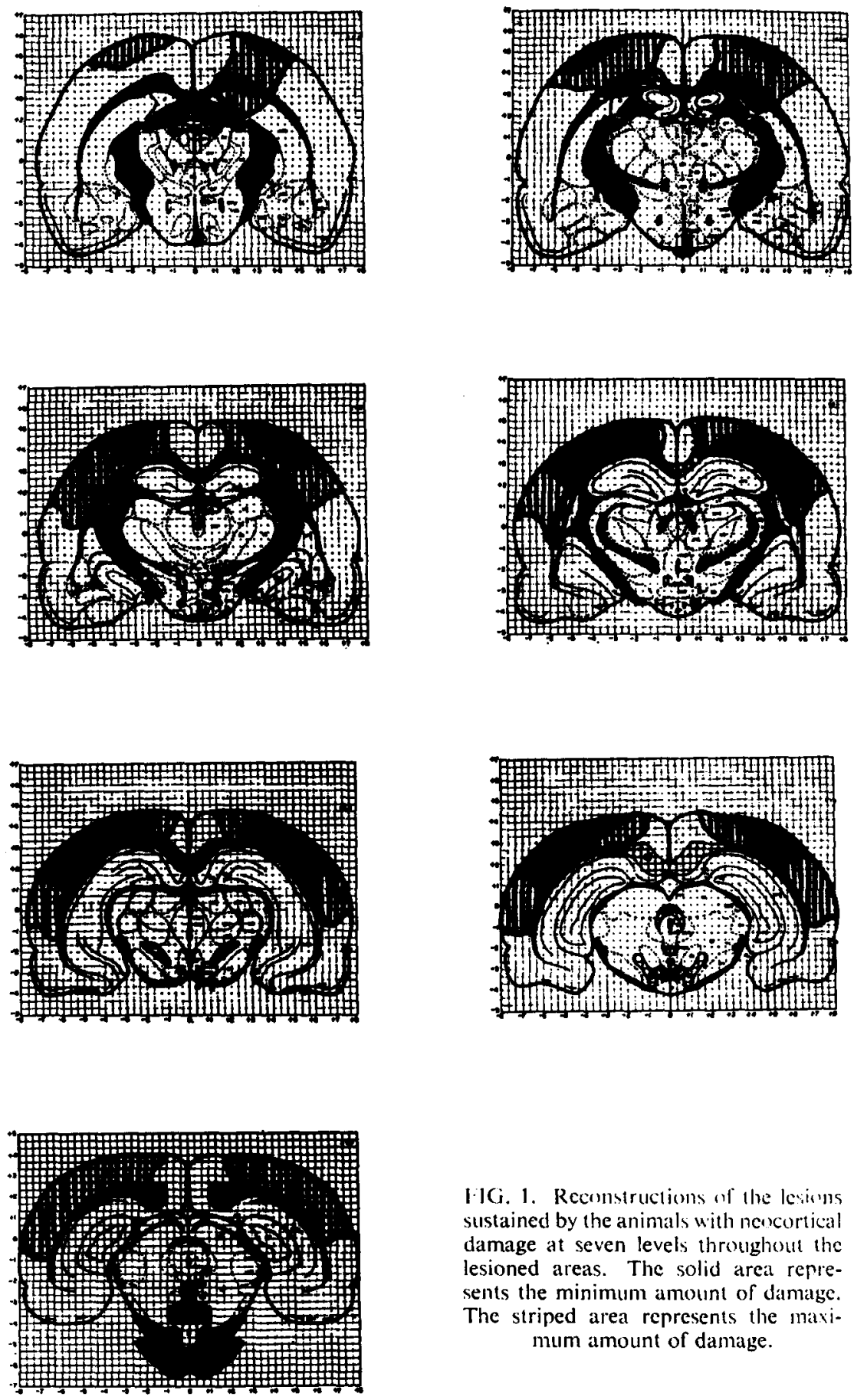

IG. 1. Reconstruclions of the lecirins sustained by the animals with noucortical damage at seven levels throughout the lesioned areas. The solid area represents the minimum amount of damage. The striped area represents the maximum amount of damage. 

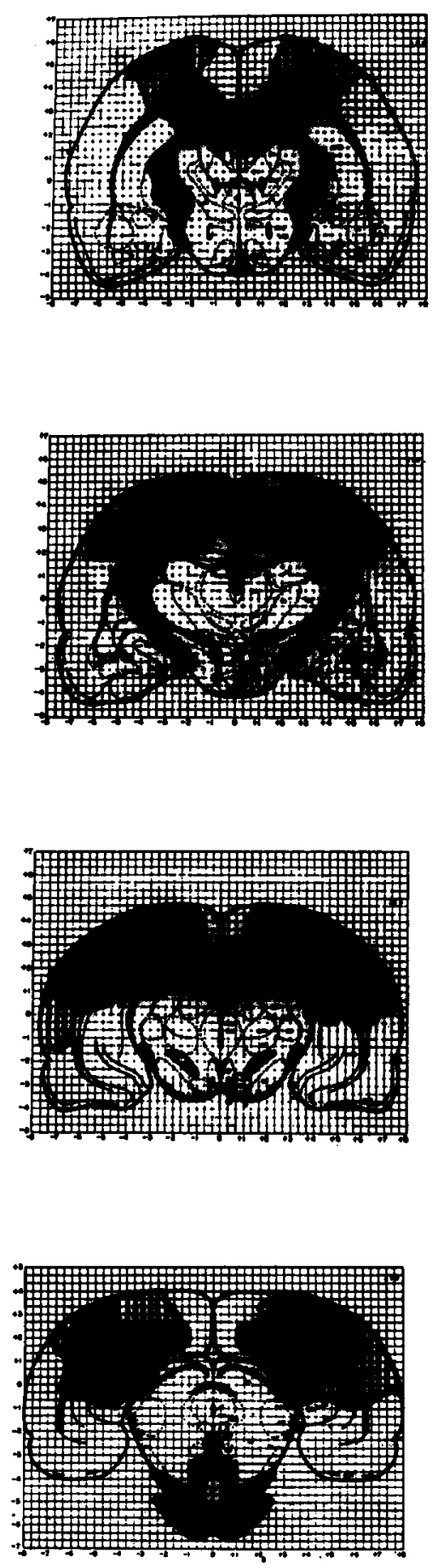
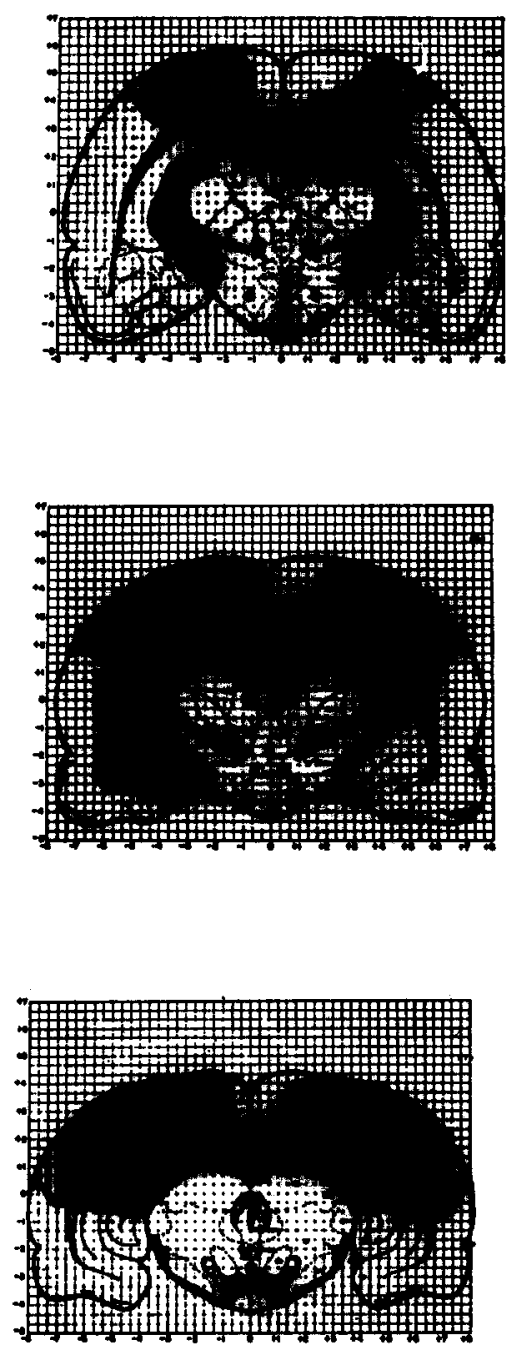

FIG. 2. Reconstructions of the lesions sustained by the animals with hippocampal damage at seven levels throughout the lesioned areas. The solid area represents the minimum amount of damage. The striped area represents the maximum amount of damage. 
the region. The animals with thalamic gliosis did not differ significantly from other lesioned animals on any of the performance measures used in the present study. In previous studies $[1,10,11]$ similar gliosis has been reported and it was not found to affect performance on a CRF or a DRL 20 schedule of reinforcement.

\section{RESULTS}

\section{CRF Performance}

All animals were given 30 daily CRF sessions of $30 \mathrm{~min}$ duration before being shifted to extinction. During this time, no clear differences were observed among the three groups of animals. A two-way analysis of variance of the data showed no effect among groups of animals, but a significant effect over CRF sessions $(F=20.45 ; p<0.01)$ and a significant interaction effect $(F=29.30 ; p<0.01)$. These results arc presented graphically in Fig. 3.

A second two-way analysis of variance was computed using the last five CRF sessions (Days 26-30). During this period, no group effect was found and no effect over sessions; however, there was a significant interaction effect $(F=10.52$; $p<0.01$ ).

On days 28-30, cumulative recordings were made of all animals' bar presses on CRF. There were no obvious differences in the recordings obtained from the three groups of animals. Consequently, a more detailed analysis was undertaken. The following measures were computed for each animal for each session: (1) latency to first bar press, (2) number of $3 \mathrm{~min}$ and $6 \mathrm{~min}$ periods of no response, and (3) number of minutes required for the animal to reach onehalf of its bar presses for the session. The three groups of animals failed to differ significantly on any of the above measures as evaluated by analyses of variance of the data combined over the 3 days.

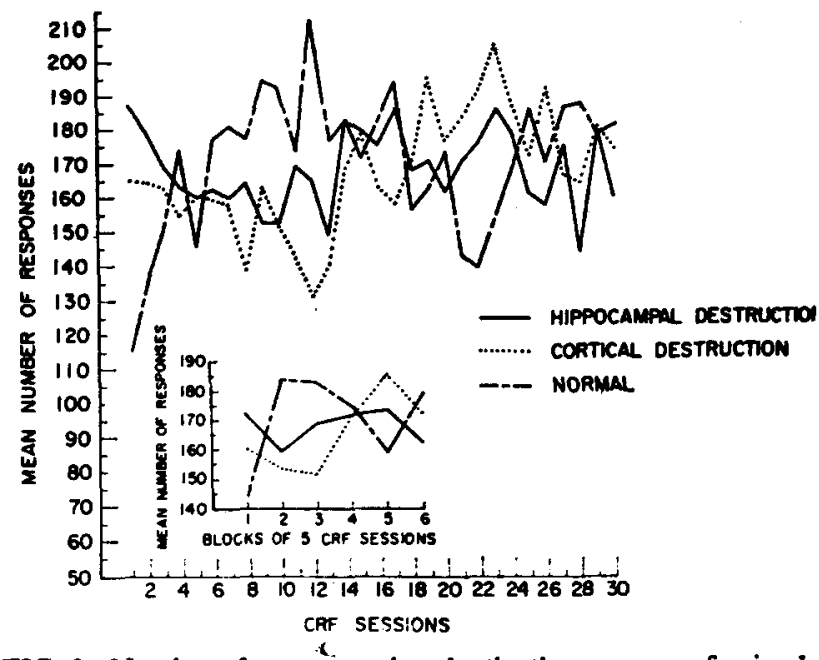

FIG. 3. Number of responses given by the three groups of animals during the continuous reinforcement sessions. The insert presents the same data plotted in terms of blocks of five continuous reinforcement sessions.

First Extinction Period: (Days 31-46)

Figure 4 shows the mean number of bar presses for the three groups of $S$ s animals for the first 16 extinction sessions (31-46). A two-way analysis of variance of these data revealed no differences among groups of animals, but a significant interaction effect $(F=11.49 ; p<0.01)$. Even though there were no overall differences in the number of bar presses emitted by the three groups of animals during sessions 31-46, the significant interaction effect indicated a more detailed

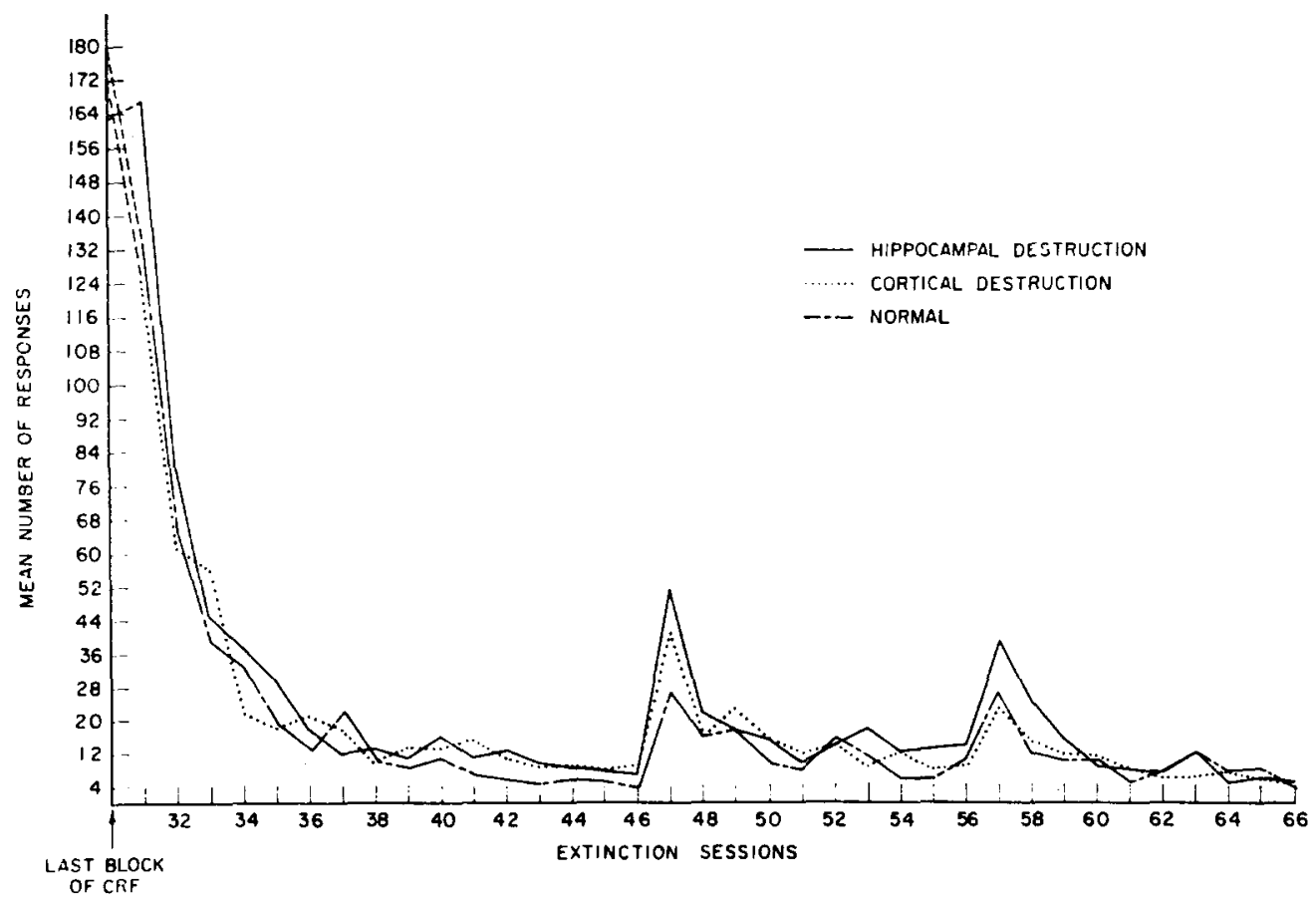

FIG. 4. Number of responses given by the three groups of animals during the extinction sessions. 
analysis should be undertaken for day 31. Using the Newman-Keuls test as described by Winer [12], it was possible to determine that the hippocampectomized rats bar pressed significantly more than the cortical control animals $(p<0.01)$ and the unoperated rats $(p<0.01)$ on day 31 . There was no statistical difference between the control and unoperated animals in number of bar presses on this same day. On each subsequent day (days 32-46), the three groups of animals did not differ among themselves (Newman-Keuls) in the number of bar presses emitted.

Inspection of the cumulative recordings for days 31-46 revealed no obvious differences among the groups of animals. A more detailed analysis of the first three extinction sessions $(31-33)$ and the last three $(44-46)$ was undertaken. The same four measures that were computed for each animal on days 28-30 were computed for each of these 6 days. Analyses of variance of all four measures combined over each three day period did not show any significant differences among the three groups of animals.

\section{Second Extinction Period: (Days 47-56)}

It may be recalled that on day 47 , one pellet was discharged into the feeding dish of the operant chamber and that the animal was allowed to experience 30 reinforced responses before the $30 \mathrm{~min}$ extinction session took place. The latency to the first bar press and the time needed to obtain the 30 reinforcements did not differ significantly (analyses of variance) among the three groups of animals. that the hippocampectomized rats $(F=61.30 ; p<0.01)$ and the neodecorticate rats $(F=50.43 ; p<0.01)$ differed significantly from the normal animals during this time. The two lesioned groups were not statistically different from each other.

On day 47 , the hippocampectomized rats (Newman-Keuls; $p<0.05$ ) and the control animals (Newman-Keuls; $p<0.05$ ) differed significantly from the unoperated rats in the number of bar presses. The two lesioned groups did not differ statistically. On each subsequent day (days 48-56), the three groups of animals did not differ among themselves (NewmanKeuls) in the number of bar presses emitted.

The same analysis of the cumulative records that was undertaken for days 31-33 and 44-46 was computed for days 47-49 and 54-56 with the same results.

\section{Third Extinction Period: (Days 57-66)}

On day 57, the same procedure was followed as on day 46. The three groups of animals did not differ significantly (analyses of variance) among themselves in the latency to first bar press or in the time needed to obtain the 30 reinforcements.

The mean number of bar presses for each day from day 57 to 66 is presented in Fig. 4. These data were subjected to a two-way analysis of variance and the groups were not found to differ among themselves; there was a significant interaction effect $(F=1.77 ; p<0.05)$. On both days 57 and 58 , the hippocampectomized rats bar pressed more than the

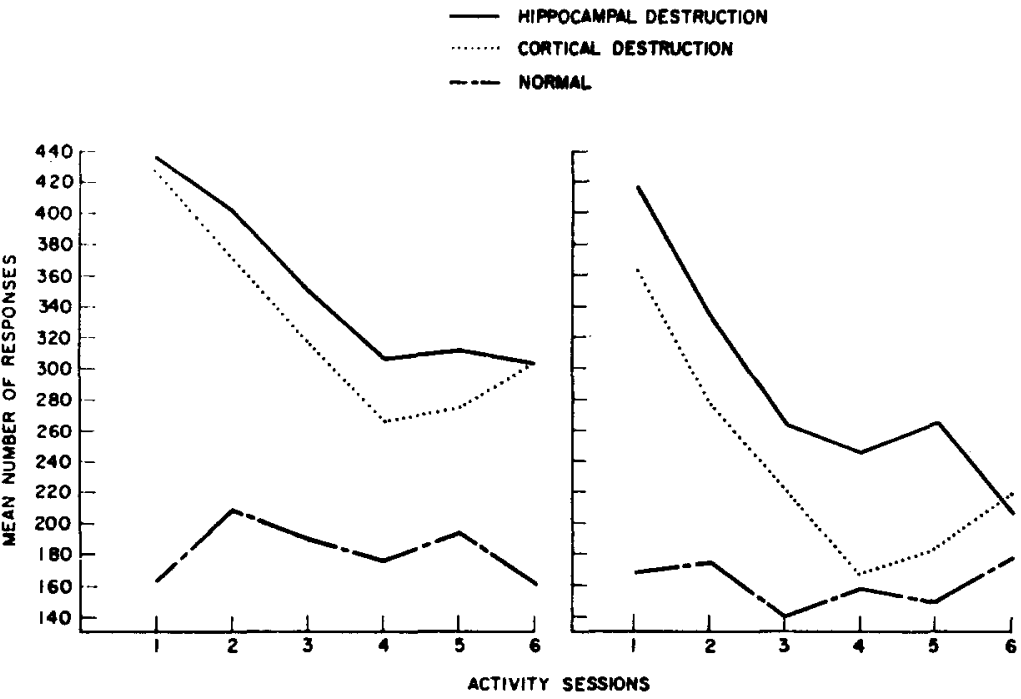

FIG. 5. Number of responses given by the three groups of animals during the sessions in the activity chamber. The figure on the left presents the data gathered $1 \mathrm{hr}$ before the animals were placed in the operant chamber; the figure on the right presents the data collected $3 \mathrm{hr}$ after the animals had eaten.

Figure 4 presents the mean number of bar presses for the three groups of animals for extinction sessions 47-56. During this period, the lesioned animals bar pressed more than the unoperated animals. A two-way analysis of variance of the data revealed a significant difference among groups of animals $(\mathrm{F}=6.53 ; p<0.01)$ and a significant interaction effect $(\mathrm{F}=10.41 ; p<0.01)$. Individual comparisons showed cortical control (Newman-Keuls; $p<0.01$ ) and the unoperated rats (Newman-Keuls; $p<0.01$ ). There was no statistical difference in bar presses between the control and unoperated rats on either day. On each subsequent day (days 59-66), the three groups of animals did not differ among themselves in total number of bar presses emitted (Newman-Keuls). 
The cumulative recordings of days 57-59 and 64-66 were subjected to the same analyses used previously with the same results.

\section{Activity Data}

On days 28-33, each subject was placed in the activity chamber for $30 \mathrm{~min}, 1 \mathrm{hr}$ before being placed in the operant chamber and for a second $30 \mathrm{~min}$ period $3 \mathrm{hr}$ after eating. Figure 5 presents the mean number of responses as reported by the electromagnetic counter for the three groups of animals during these sessions.

Figure 5 (left) presents the data for the six sessions $1 \mathrm{hr}$ before the sessions in the operant chamber. A two-way analysis of variance showed a significant effect among groups of subjects $(F=32.45 ; p<0.01)$, a significant sessions effect $(F=9.25 ; p<0.01)$, and a significant interaction effect $(F=2.65 ; p<0.01)$. Individual comparisons revealed that the hippocampectomized rats $(F=5.33 ; p<0.05)$ and the control animals $(F=4.93 ; p<0.05)$ differed from the unoperated rats. There was no difference between the lesioned groups.

Figure 5 (right) represents the data collected three hr after the completion of eating. A two-way analysis of variance failed to reveal a significant group effect, but there was a significant effect over sessions $(F=8.24 ; p<0.01)$, and a significant interaction effect $(F=2.11 ; p<0.05)$.

There were no obvious differences in the cumulative recordings of the activity sessions for the three groups of rats. Again, a more detailed analysis was undertaken. For every animal the following measures were computed for each session: number of 2, 4, and 6-min periods of no responses and number of minutes to reach one-fourth, one-half, and threefourths of the total number of responses for the period. The three groups of animals failed to differ significantly on any of the above measures as evaluated by analyses of variance of the data combined separately for each set of six activity sessions.

\section{Lesion Characteristics}

None of the various response measures reported for the animals while on CRF, extinction, or during the activity sessions were found to be systematically related to total brain damage or damage to specific structures. Within each of the lesioned groups, each response measure was correlated (Pearson product-moment) with total brain damage and damage to the structures listed in Table 1 . None of the correlations were found to approach statistical significance. Also, site of lesion was not found to be related to the performance measures within either lesioned group.

\section{DIsCUssion}

The outcomes of this experiment are of special importance for the interpretation of other experiments with animals with hippocampal destruction. In previous work, hippocampal destruction had been found to influence subjects in such a way that when a change from continuous reinforcement to intermittant reinforcement occurred, their response rates increased, and remained higher, than those of animals with only neocortical lesions under the same conditions $[1,6,10$, 11]. Evaluating these previous studies in light of our present results leads to the conclusion that hippocampal destruction does not necessarily lead to an increased resistance to extinction. Apparently, there are certain manipulations of the experimental procedures which can produce "perseverative behaviors." In the runway situation, these involve alterations in the intertrial interval $[5,7]$; while in the operant situation, the change from continuous reinforcement schedules to intermittant schedules seems to be essential. Continuous schedules of reinforcement, whether of acquisition or extinction, do not affect the animals so as to produce permanent heightened response rates.

A point which should be emphasized is that while we have used the term "response perseveration" in discussing the results from this experiment, and from others, the changes produced by hippocampal destruction are not simply a perseveration of responsiveness or a failure of inhibition. After the shift to an intermittant schedule, response rates do not maintain themselves at pre-existing levels. There is a substantial, if not dramatic, increase in rate, and this implies that the behavioral change produced by the lesion is more than a failure to suppress previous rates. It represents an exaggerated responsiveness to the change in reinforcement contingencies. As mentioned above, the releasing characteristic for this increased responsiveness may be the uncertainty of the intermittant schedules when occurring after a continuous schedule.

Recently, Klüver [8] has proposed that the temporal lobe limbic areas are important for an animal's coping with "shifting and fluctuating phenomena" of the environment, and such an interpretation would be entirely in accord with our present results. Three times during the course of this study the animals were subjected to "shifting and fluctuating phenomena." This occurred on days 31,47 , and 57 . On all three of these occasions the hippocampectomized rats bar pressed significantly more than the unoperated animals. They also responded more than the cortical control animals on these three occasions. It must be mentioned however, that on one of the days (day 47) the difference was not great enough to be statistically significant.

The finding that the lesioned animals were more active than the unoperated subjects $1 \mathrm{hr}$ before the sessions in the operant chamber, but not $3 \mathrm{hr}$ after eating, suggests that the hours of food deprivation might have had differential effects on the lesioned and non-lesioned animals. Douglas and Isaacson [3] measured the activity levels of rats with hippocampal destruction in an apparatus somewhat similar to the one used in the present study and found that these animals were more active than cortical control or unoperated subjects. All animals were, however, tested under ad libitum food conditions. The prospect that hours of food deprivation affect activity levels of lesioned and non-lesioned subjects differentially is an interesting one which awaits further research. 


\section{REFERENCES}

1. Clark, C. V. H. and R. L. Isaacson. Effect of bilateral hippocampal ablation on DRL performance. J. comp. physiol. Psychol. 59: 137-140, 1965.

2. DeGroot, J. The Rat Forebrain in Stereotaxic Coordinates. Amsterdam: N. V. Noord-Hollandsche Uitgevers Maatschappij, 1959.

3. Douglas, R. J. and R. L. Isaacson. Hippocampal lesions and activity. Psychon. Sci. 1: 187-188, 1964.

4. Isaacson, R. L., R. J. Douglas and R. Y. Moore. The effect of radical hippocampal ablation on acquisition of avoidance responses. J. comp. physiol. Psychol. 54: 625-628, 1961.

5. Jarrard, L. E., R. L. Isaacson and W. O. Wickelgren. Effects of hippocampal ablation and intertrial interval on runway acquisition and extinction. J. comp. physiol. Psychol. 57: 442-444, 1964.

6. Jarrard, L. E. Hippocampal ablation and operant behavior in the rat. Psychon. Sci. 2: 115-116, 1965.
7. Jarrard, L. E. and R. L. Isaacson. Hippocampal ablation in rats: effects of intertrial interval. Nature, Lond. 207: 109. $110,1965$.

8. Klüver, H. Neurobiology of normal and abnormal perception. In: Psychopathology of Perception, edited by P. H. Hoch and J. Zubin. New York: Grune and Stratton, 1965.

9. Peacock, L. J. and M. Williams. An ultrasonic device for recording activity. Am. J. Psychol. 75: 648-652, 1962.

10. Schmaltz, L. W. and R. L. Isaacson. The effects of preliminary training conditions upon DRL 20 performance in the hippocampectomized rat. Physiol. Behav. 1: 175-182, 1966.

11. Schmaltz, L. W. and R. L. Isaacson. Postoperative retention of a DRL 20 schedule by hippocampectomized and partially neodecorticate rats. J. comp. physiol. Psychol. 62: 128-132, 1966.

12. Winer, B. J. Statistical Principles in Experimental Design. New York: McGraw-Hill, 1962. 